\title{
SHARP INEQUALITIES FOR LOGARITHMIC COEFFICIENTS AND THEIR APPLICATIONS
}

\author{
S. PONNUSAMY AND TOSHIYUKI SUGAWA
}

\begin{abstract}
I. M. Milin proposed, in his 1971 paper, a system of inequalities for the logarithmic coefficients of normalized univalent functions on the unit disk of the complex plane. This is known as the Lebedev-Milin conjecture and implies the Robertson conjecture which in turn implies the Bieberbach conjecture. In 1984, Louis de Branges settled the long-standing Bieberbach conjecture by showing the Lebedev-Milin conjecture. Recently, O. Roth proved an interesting sharp inequality for the logarithmic coefficients based on the proof by de Branges. In this paper, following Roth's ideas, we will show more general sharp inequalities with convex sequences as weight functions and then establish several consequences of them. We also consider the inequality with the help of de Branges system of linear ODE for non-convex sequences where the proof is partly assisted by computer. Also, we apply some of those inequalities to improve previously known results.
\end{abstract}

\section{Estimates OF LOGARITHMiC COEFFICIENTS}

Let $\mathcal{A}$ denote the set of normalized analytic functions on the open unit disk $\mathbb{D}=\{z \in$ $\mathbb{C}:|z|<1\}$ and $\mathcal{S}$ denote its subclass of univalent functions. We define the logarithmic coefficients of $f$ by the formula

$$
\log \frac{f(z)}{z}=2 \sum_{n=1}^{\infty} \gamma_{n} z^{n} .
$$

Throughout the discussion, $\gamma_{n}:=\gamma_{n}(f)$ denote the logarithmic coefficients of a function $f \in \mathcal{S}$. Louis de Branges [5] solved the long-standing Bieberbach conjecture by showing the Lebedev-Milin conjecture (see also [7]): For each $n \geq 1$,

$$
\sum_{k=1}^{n} k(n-k+1)\left|\gamma_{n}\right|^{2} \leq \sum_{k=1}^{n} \frac{n-k+1}{k},
$$

where equality holds if and only if $f$ is the Koebe function $K(z)=z /(1-z)^{2}$ or its rotation $e^{-i \theta} K\left(e^{i \theta} z\right)=z /\left(1-e^{i \theta} z\right)^{2}$ for some $\theta \in \mathbb{R}$. Note that for $f(z)=z /\left(1-e^{i \theta} z\right)^{2}$ we have $\gamma_{n}=e^{i n \theta} / n$ for $n=1,2, \ldots$

As an application of the de Branges theorem (1.2), we will show a more general inequality. As a preparation, we recall a notion of convexity for sequences. A sequence of real numbers $p_{n}, n=1,2,3, \ldots$, is called convex if $p_{n}-2 p_{n+1}+p_{n+2} \geq 0$ for all $n \geq 1$.

2010 Mathematics Subject Classification. Primary 30C50; Secondary 30C75.

Key words and phrases. logarithmic coefficient, Milin conjecture, de Branges theorem.

The present research was supported by JSPS Grant-in-Aid for Scientific Research (B) 22340025 and JP17H02847. The work of the first author is supported by Mathematical Research Impact Centric Support (MATRICS) of DST, India (MTR/2017/000367). 
Note that $p_{n}=\varphi(n), n=1,2,3, \ldots$, form a convex sequence if $\varphi(x)$ is a convex function on $[1,+\infty)$ in the ordinary sense. We can now state it as follows.

Theorem 1.1. Let $p_{n}, n=1,2,3, \ldots$, be a convex sequence of non-negative numbers with $p_{1}>0$ such that $\sum_{n=1}^{\infty}\left(p_{n} / n\right)<+\infty$. For $f \in \mathcal{S}$ with expansion (1.1), the inequality

$$
\sum_{n=1}^{\infty} n p_{n}\left|\gamma_{n}\right|^{2} \leq \sum_{n=1}^{\infty} \frac{p_{n}}{n}
$$

holds. Moreover, the inequality is strict unless $f(z)$ has the form $z /\left(1-e^{i \theta} z\right)^{2}$ for some $\theta \in \mathbb{R}$.

We remark that the theorem is not really new. The same statement was already made by de Branges [5] when the convex sequence $p_{n}$ is eventually vanishing, i.e., $p_{n}=0$ for sufficiently large numbers $n$. Zemyan in his 1993 paper [16] extended it to general convex sequences by approximating them with eventually vanishing ones. Therefore, he did not provide equality conditions. For convenience of the reader, we give a direct proof of the theorem.

Proof of Theorem 1.1. First note that $p_{n} / n \rightarrow 0$ as $n \rightarrow \infty$ by the convergence assumption. Put $q_{n}=p_{n}-p_{n+1}$ and

$$
\lambda_{n}=q_{n}-q_{n+1}=p_{n}-2 p_{n+1}+p_{n+2}
$$

for $n=1,2,3, \ldots$ Then, by convexity, $\lambda_{n} \geq 0$ and thus $q_{n}$ is a non-increasing sequence. In particular, $q_{n}$ has a limit, say $q$, as $n \rightarrow \infty$. If $q \neq 0$, then $p_{n}$ is asymptotically equal to $n q$, which violates $p_{n} / n \rightarrow 0$. Hence, we conclude that $q=0$. Since $q_{n}$ is non-increasing, we have $p_{n}-p_{n+1}=q_{n} \geq 0$, which means $p_{n}$ is non-increasing. In particular, $p_{n}$ has a limit, say $p$, as $n \rightarrow \infty$. Since $p_{n} \geq 0$, we have $p \geq 0$. If $p>0$, then $p_{n} \geq p$, which implies $\sum p_{n} / n \geq \sum p / n=+\infty$, a contradiction. Hence, the convergence assumption forces the sequence $p_{n}$ to converge to 0 . Here we also note that, by the assumption $p_{1}>0$, there is an $n \geq 1$ such that $\lambda_{n}>0$.

We now sum up the inequalities (1.2) with the weight $\lambda_{n} \geq 0$ to obtain

$$
\sum_{n=1}^{\infty} \lambda_{n} \sum_{k=1}^{n} k(n-k+1)\left|\gamma_{k}\right|^{2} \leq \sum_{n=1}^{\infty} \lambda_{n} \sum_{k=1}^{n} \frac{n-k+1}{k} .
$$

Here, we note that equality holds in (1.4) if and only if $f(z)=z /\left(1-e^{i \theta} z\right)^{2}$, because equality must hold in (1.2) for at least one $n$. The interchange of the order of summation gives us the inequality

$$
\sum_{k=1}^{\infty} k\left|\gamma_{k}\right|^{2} \sum_{n=k}^{\infty} \lambda_{n}(n-k+1) \leq \sum_{k=1}^{\infty} \frac{1}{k} \sum_{n=k}^{\infty} \lambda_{n}(n-k+1) .
$$

If

$$
p_{k}=\sum_{n=k}^{\infty} \lambda_{n}(n-k+1), \quad k \geq 1,
$$


then we would have the inequality (1.3). We now show (1.5) . Since $p_{n} \rightarrow 0$, we have

$$
p_{k}=\sum_{n=k}^{\infty}\left(p_{n}-p_{n+1}\right)=\sum_{n=k}^{\infty} q_{n} .
$$

For convenience, for a fixed $k \geq 1$, we put $s_{n}=n-k$ for $n=k, k+1, \ldots$ Letting $N \geq k$, we compute

$$
\begin{aligned}
p_{k}-p_{N+1} & =\sum_{n=k}^{N} q_{n}=\sum_{n=k}^{N}\left(s_{n+1}-s_{n}\right) q_{n} \\
& =s_{N+1} q_{N}-s_{k} q_{k}+\sum_{n=k}^{N-1} s_{n+1}\left(q_{n}-q_{n+1}\right) \\
& =s_{N+1} q_{N}+\sum_{n=k}^{N-1} s_{n+1} \lambda_{n} .
\end{aligned}
$$

Here, we used the fact that $s_{k}=0$. In particular, we have

$$
s_{N+1} q_{N}+\sum_{n=k}^{N-1} s_{n+1} \lambda_{n} \leq p_{k} .
$$

Since each term in the left-hand side is non-negative,

$$
\sum_{n=k}^{\infty} s_{n+1} \lambda_{n}=\sup _{N>k} \sum_{n=k}^{N-1} s_{n+1} \lambda_{n} \leq p_{k}<+\infty .
$$

Recalling $p_{N+1} \rightarrow 0$, we see by (1.6) that $s_{N+1} q_{N}$ also has a limit, say $b$, as $N \rightarrow \infty$. If $b \neq 0$, then $q_{n}$ is asymptotically $b / n$ and thus $p_{n}$ is asymptotically $b \log n$, which contradicts $p_{n} \rightarrow 0$. Thus we conclude that $b=0$. Letting $N \rightarrow \infty$ in (1.6), we obtain the relation

$$
p_{k}=\sum_{n=k}^{\infty} s_{n+1} \lambda_{n}=\sum_{n=k}^{\infty}(n-k+1) \lambda_{n}
$$

and hence (1.5) is proved.

In a recent paper by Roth [15], he made the nice observation that (1.4) could hold even if some of $\lambda_{n}$ are negative. His idea is to show the inequality

$$
\sum_{n=1}^{N} \lambda_{n} \sum_{k=1}^{n} k(n-k+1)\left|\gamma_{k}\right|^{2} \leq \sum_{n=1}^{N} \lambda_{n} \sum_{k=1}^{n} \frac{n-k+1}{k}
$$

for some $N \geq 2$ by using the original idea of de Branges. If $\lambda_{n} \geq 0$ for $n>N$, we obtain (1.4) by summing up for $n>N$ with weight $\lambda_{n}$. We will take a closer look at this case in the third section. 


\section{Consequences of Theorem 1.1}

By various choices of positive convex sequences $p_{n}$, we obtain many sharp inequalities on the logarithmic coefficients $\gamma_{n}$ of $f \in \mathcal{S}$. The most fundamental one is perhaps $p_{n}=r^{2 n}$ for a positive number $r$. It is easy to check that this sequence satisfies the assumptions of Theorem 1.1 if and only if $r<1$. Then we obtain the sharp inequality for the logarithmic area

$$
\sum_{n=1}^{\infty} n\left|\gamma_{n}\right|^{2} r^{2 n} \leq \sum_{n=1}^{\infty} \frac{r^{2 n}}{n}=\log \frac{1}{1-r^{2}}
$$

which is known as the Bazilevic conjecture and proved by Milin and Grinshpan [10] (see also [9]). The next fundamental example is $p_{n}=n^{-\alpha}$ for a constant $\alpha>0$. Since $\varphi(x)=x^{-\alpha}$ is convex on $x>0$, the sequence $p_{n}=\varphi(n)$ is convex. Therefore, as a corollary of Theorem 1.1, we obtain the inequality

$$
\sum_{n=1}^{\infty} n^{1-\alpha}\left|\gamma_{n}\right|^{2} \leq \sum_{n=1}^{\infty} \frac{1}{n^{1+\alpha}}=\zeta(\alpha+1),
$$

where $\zeta(x)$ denotes the Riemann zeta function. Equality holds if and only if $f$ is a rotation of the Koebe function $z /(1-z)^{2}$. This inequality was proved by Zemyan [16, Theorem 3 (b)]. Letting $\alpha=1$ in particular, we obtain the Duren-Leung inequality [6]

$$
\sum_{n=1}^{\infty}\left|\gamma_{n}\right|^{2} \leq \frac{\pi^{2}}{6}
$$

It is worth recalling that this inequality was proved even before de Branges' proof of the Lebedev-Milin conjecture.

We summarize other choices in the following lemma.

Lemma 2.1. For each choice of the following, the sequence $p_{n}(n=1,2,3, \ldots)$ is positive and convex.

(1) $p_{n}=\frac{1}{n+\alpha}$ and $\alpha>-1$,

(2) $p_{n}=\frac{n}{n^{2}+a n+b}$ for $a, b \in \mathbb{R}$ with $a+b+1>0, a+3 \geq 0$ and $(6+a) b \leq 6$.

(3) $p_{n}=\frac{n}{(n+\alpha)(n+\beta)}$ for $\alpha>-1, \beta>-1$ with $(\alpha+\beta+6) \alpha \beta \leq 6$ and $\alpha \beta \leq 6$.

(4) $p_{n}=\frac{1}{n^{2}+a n+b}$ for $a, b \in \mathbb{R}$ with $a+b+1>0, a+2 \geq 0$ and $b \leq a^{2}+6 a+11$.

(5) $p_{n}=\frac{1}{(n+\alpha)(n+\beta)}$ for $\alpha>-1, \beta>-1$.

(6) $p_{n}=\frac{n^{2}}{(n+\alpha)^{2}(n+\beta)}$ for $\alpha>-1, \beta>-1$ with $|\alpha|\left(1+3|\beta|+\beta^{2}\right) \leq 1 / 2$.

(7) $p_{n}=(n+\alpha) r^{n}$ for $\alpha>-1$ and $r \in(0,1)$ with $2 \leq(\alpha+1) \log (1 / r)$.

Proof. We will take the following strategy to show the assertion. First we choose a smooth function $\varphi$ so that $p_{n}=\varphi(n)$. If we confirm that $\varphi(x)$ is convex on $N \leq x<+\infty$ for an 
integer $N \geq 1$, then it is enough to check the condition $\lambda_{n}=p_{n}-2 p_{n+1}+p_{n+2} \geq 0$ for $n=1,2, \ldots, N-1$.

(1) Since $\varphi(x)=1 /(x+\alpha)$ is convex on $1 \leq x$ for $\alpha>-1$, the assertion follows.

(2) First note that $p_{n}>0$ for $n \geq 1$ by the first two conditions on parameters. Indeed, $n^{2}+a n+b=(n-1)(n+1+a)+1+a+b \geq 1+a+b>0$ for $n \geq 1$. As a necessary condition, we have

$$
\lambda_{1}=\frac{2(6-6 b-a b)}{(1+a+b)(4+2 a+b)(9+3 a+b)} \geq 0,
$$

which is certainly implied by the assumption. Let $\varphi(x)=x /\left(x^{2}+a x+b\right)$ and compute

$$
\varphi^{\prime \prime}(x)=\frac{2\left(x^{3}-3 b x-a b\right)}{\left(x^{2}+a x+b\right)^{3}} .
$$

We note here that $b \leq 6 /(6+a) \leq 2$ by the assumptions $a+3 \geq 0$ and $(6+a) b \leq 6$. Since

$$
x^{3}-3 b x-a b=x^{3}-3 b x+6 b-6+(6-6 b-a b) \geq x^{3}-3 b x+6 b-6=: h(x),
$$

it is enough to show that $h(x) \geq 0$ for $x \geq 2$. Since $h^{\prime}(x)=3\left(x^{2}-b\right)$, the function $h(x)$ is increasing in $2 \leq x<+\infty$ and thus $h(x) \geq h(2)=2>0$ as required.

(3) We apply the previous case for $a=\alpha+\beta$ and $b=\alpha \beta$ to get the assertion.

(4) As in the case (2), we see that $p_{n}>0$ by the first two conditions on $a, b$. Also, the inequality

$$
\lambda_{1}=\frac{2\left(a^{2}+6 a+11-b\right)}{(1+a+b)(4+2 a+b)(9+3 a+b)} \geq 0
$$

holds by assumption. Let $\varphi(x)=1 /\left(x^{2}+a x+b\right)$ and compute

$$
\varphi^{\prime \prime}(x)=\frac{2\left(3 x^{2}+3 a x+a^{2}-b\right)}{\left.x^{2}+a x+b\right)^{3}} .
$$

Since $h(x)=3 x^{2}+3 a x+a^{2}-b$ is increasing in $x \geq-a / 2(\leq 1)$, we obtain $h(x) \geq h(2)=$ $a^{2}+6 a+12-b \geq 1>0$ for $x \geq 2$. Thus we conclude that $\varphi(x)$ is convex on $2 \leq x<+\infty$.

(5) Just apply (4) with $a=\alpha+\beta$ and $b=\alpha \beta$.

(6) Let $\varphi(x)=x^{2} /\left[(x+\alpha)^{2}(x+\beta)\right]$. Then

$$
\varphi^{\prime}(x)=\frac{2\left(x^{4}-2 \alpha x^{3}-6 \alpha \beta x^{2}-2 \alpha \beta^{2}+\alpha^{2} \beta^{2}\right.}{(x+\alpha)^{4}(x+\beta)^{3}} .
$$

For $x \geq 1$, we have

$$
x^{4}-2 \alpha x^{3}-6 \alpha \beta x^{2}-2 \alpha \beta^{2}+\alpha^{2} \beta^{2} \geq x^{4}\left(1-2|\alpha|-6|\alpha \beta|-2\left|\alpha \beta^{2}\right|\right) \geq 0,
$$

which implies that $\varphi(x)$ is convex on $1 \leq x<+\infty$.

(7) It is enough to observe the formula $\varphi^{\prime \prime}(x)=\{2+(\alpha+x)\} r^{x} \log r$ for $\varphi(x)=$ $(x+\alpha) r^{x}$.

Corollary 2.2. For the logarithmic coefficients $\gamma_{n}$ of $f \in \mathcal{S}$, the following inequalities hold. Each of them is strict unless $f$ is not a rotation of the Koebe function $z /(1-z)^{2}$. 
(1) $\sum_{n=1}^{\infty} \frac{n}{n+\alpha}\left|\gamma_{n}\right|^{2} \leq \sum_{n=1}^{\infty} \frac{1}{n(n+\alpha)}=:$ A for $\alpha>-1$. When $\alpha \neq 0$, we have the expressions

$$
A_{\alpha}=\frac{1}{\alpha} \int_{0}^{1} \frac{1-t^{\alpha}}{1-t} d t=\frac{\psi(\alpha+1)-\psi(1)}{\alpha}
$$

Here and in the sequel $\psi(x)=\Gamma^{\prime}(x) / \Gamma(x)$ denotes the Digamma function. In particular, letting $\alpha=1,2,3,1 / 2,-1 / 2$, the following sharp inequalities are deduced:

$$
\begin{aligned}
& \text { [a] } \sum_{n=1}^{\infty} \frac{n}{n+1}\left|\gamma_{n}\right|^{2} \leq 1, \\
& \text { [b] } \sum_{\substack{n=1 \\
\infty}}^{\infty} \frac{n}{n+2}\left|\gamma_{n}\right|^{2} \leq \frac{3}{4}, \\
& \text { [c] } \sum_{\substack{\infty=1 \\
\infty}}^{\infty} \frac{n}{n+3}\left|\gamma_{n}\right|^{2} \leq \frac{11}{18}, \\
& \text { [d] } \sum_{n=1}^{\infty} \frac{n}{2 n+1}\left|\gamma_{n}\right|^{2} \leq 2(1-\log 2), \\
& \text { [e] } \sum_{n=1}^{\infty} \frac{n}{2 n-1}\left|\gamma_{n}\right|^{2} \leq 2 \log 2 .
\end{aligned}
$$

(2) $\sum_{n=1}^{\infty} \frac{n^{2}}{n^{2}+\alpha^{2}}\left|\gamma_{n}\right|^{2} \leq B_{\alpha}:=\frac{\pi \alpha \operatorname{coth} \pi \alpha-1}{2 \alpha^{2}}$ for $\alpha \in(0,1]$.

(3) $\sum_{n=1}^{\infty} \frac{n^{2}}{(n+\alpha)(n+\beta)}\left|\gamma_{n}\right|^{2} \leq C_{\alpha, \beta}$ for $\alpha, \beta \in(-1,+\infty)$ with $(\alpha+\beta+6) \alpha \beta \leq 6$ and $\alpha \beta \leq 6$. Here,

$$
C_{\alpha, \beta}= \begin{cases}\frac{1}{\beta-\alpha} \int_{0}^{1} \frac{t^{\alpha}-t^{\beta}}{1-t} d t=\frac{\psi(1+\beta)-\psi(1+\alpha)}{\beta-\alpha} & \text { if } \alpha \neq \beta \\ \sum_{n=1}^{\infty} \frac{1}{(n+\alpha)^{2}}=\int_{0}^{1} \frac{t^{\alpha} \log (1 / t)}{1-t} d t=\psi^{\prime}(1+\alpha), & \text { if } \alpha=\beta .\end{cases}
$$

In particular,

$$
\begin{aligned}
& \text { [a] } \sum_{n=1}^{\infty} \frac{n^{2}}{4 n^{2}-1}\left|\gamma_{n}\right|^{2} \leq \frac{1}{2} \\
& \text { [b] } \sum_{n=1}^{\infty} \frac{n^{2}}{(n+1)(2 n+1)}\left|\gamma_{n}\right|^{2} \leq 2 \log 2-1 .
\end{aligned}
$$

(4) $\sum_{n=1}^{\infty} \frac{n}{(n+\alpha)(n+\beta)}\left|\gamma_{n}\right|^{2} \leq D_{\alpha, \beta}$ for $\alpha, \beta \in(-1,+\infty)$, where

$$
D_{\alpha, \beta}=\int_{0}^{1} \frac{\beta\left(1-t^{\alpha}\right)-\alpha\left(1-t^{\beta}\right)}{\alpha \beta(\beta-\alpha)(1-t)} d t=-\frac{1}{\beta-\alpha}\left(\frac{\psi(1+\beta)}{\beta}-\frac{\psi(1+\alpha)}{\alpha}\right)-\frac{\psi(1)}{\alpha \beta}
$$


for nonzero $\alpha, \beta$ with $\alpha \neq \beta$,

$$
D_{\alpha, 0}=D_{0, \alpha}=\frac{\zeta(2)-A_{\alpha}}{\alpha}=\frac{\pi^{2} / 6-A_{\alpha}}{\alpha}
$$

and

$$
D_{\alpha, \alpha}=\int_{0}^{1} \frac{1-t^{\alpha}-\alpha t^{\alpha} \log (1 / t)}{\alpha^{2}(1-t)} d t=\frac{\psi(1+\alpha)-\psi(1)}{\alpha^{2}}-\frac{\psi^{\prime}(1+\alpha)}{\alpha}
$$

for nonzero $\alpha$, and

$$
D_{0,0}=\zeta(3) \text {. }
$$

In particular,

$$
\begin{aligned}
& \text { [a] } \sum_{n=1}^{\infty} \frac{n}{(n+1)(n+2)}\left|\gamma_{n}\right|^{2} \leq \frac{1}{4} . \\
& \text { [b] } \sum_{\substack{n=1 \\
n}}^{\infty} \frac{1}{n+1}\left|\gamma_{n}\right|^{2} \leq \frac{\pi^{2}}{6}-1, \\
& \text { [c] } \sum_{n=1}^{\infty} \frac{n}{(n+1)^{2}}\left|\gamma_{n}\right|^{2} \leq 2-\frac{\pi^{2}}{6} .
\end{aligned}
$$

(5) $\sum_{n=1}^{\infty} \frac{n^{3}}{(n+\alpha)^{2}(n+\beta)}\left|\gamma_{n}\right|^{2} \leq E_{\alpha, \beta}$ for $\alpha, \beta \neq 0$ with $|\alpha|\left(1+3|\beta|+\beta^{2}\right) \leq 1 / 2$, where

$$
E_{\alpha, \beta}= \begin{cases}\frac{\beta C_{\alpha, \beta}-\alpha C_{\alpha, \alpha}}{\beta-\alpha} & \text { if } \alpha \neq \beta, \\ \psi^{\prime}(1+\alpha)+\frac{\alpha}{2} \psi^{\prime \prime}(1+\alpha) & \text { if } \alpha=\beta .\end{cases}
$$

(6) $\sum_{n=1}^{\infty} n(n+\alpha)\left|\gamma_{n}\right|^{2} r^{2 n} \leq \frac{r^{2}}{1-r^{2}}+\alpha \log \frac{1}{1-r^{2}}$ for $\alpha>-1$ and $0<r<1$ with $1 \leq(\alpha+1) \log (1 / r)$.

Proof. Basically, all the inequalities follow from Theorem 1.1 and Lemma 2.1. The remaining task is only to compute the sum $\sum_{n=1}^{\infty} p_{n} / n$.

(1) By the formula

$$
A_{\alpha}=\sum_{n=1}^{\infty} \frac{1}{n(n+\alpha)}=\frac{1}{\alpha} \sum_{n=1}^{\infty}\left(\frac{1}{n}-\frac{1}{n+\alpha}\right)=\frac{1}{\alpha} \sum_{n=1}^{\infty} \int_{0}^{1}\left(t^{n-1}-t^{n+\alpha-1}\right) d t
$$

we easily obtain the first expression. The second expression can be obtained by the well-known formula (see [1, 6.3.16])

$$
\psi(1+x)=-\gamma+\sum_{n=1}^{\infty} \frac{x}{n(n+x)}=-\gamma+\sum_{n=1}^{\infty}\left(\frac{1}{n}-\frac{1}{n+x}\right) \quad(x \neq-1,-2,-3, \cdots),
$$

where $\gamma$ is Euler's constant. The following formulae are convenient in practical computations:

$$
\psi(1+x)=\psi(x)+\frac{1}{x} \text { and } \psi(1)=-\gamma
$$


(2) We need to show the identity

$$
\sum_{n=1}^{\infty} \frac{1}{n^{2}+\alpha^{2}}=\frac{\pi \alpha \operatorname{coth} \pi \alpha-1}{2 \alpha^{2}}
$$

This can be deduced by subsituting $z=i \alpha$ into the well-known formula (see [2, p. 189])

$$
\pi \cot \pi z=\frac{1}{z}+\sum_{n=1}^{\infty} \frac{2 z}{z^{2}-n^{2}}
$$

(3) The required formula

$$
C_{\alpha, \beta}=\sum_{n=1}^{\infty} \frac{1}{(n+\alpha)(n+\beta)}
$$

can be shown in the same way as in (2). The particular cases follow from the computations $C_{1 / 2,-1 / 2}=2$ and $C_{1 / 2,1}=2(2 \log 2-1)$.

(4) We need to check the formula

$$
D_{\alpha, \beta}=\sum_{n=1}^{\infty} \frac{1}{n(n+\alpha)(n+\beta)} .
$$

For the generic case $\alpha \neq \beta$, we may write the right-hand side in the form

$$
\frac{1}{\beta-\alpha}\left(\sum_{n=1}^{\infty} \frac{1}{n(n+\alpha)}-\sum_{n=1}^{\infty} \frac{1}{n(n+\beta)}\right)=-\left(\frac{A_{\beta}-A_{\alpha}}{\beta-\alpha}\right)
$$

and the assertion follows immediately from Case (2). The rest of the assertions follows easily from a standard limiting process.

(5) We have only to use the expression

$$
\frac{n}{(n+\alpha)^{2}(n+\beta)}=\frac{\beta}{\beta-\alpha} \frac{1}{(n+\alpha)(n+\beta)}-\frac{\alpha}{\beta-\alpha} \frac{1}{(n+\alpha)^{2}}
$$

for $\alpha \neq \beta$. The case when $\alpha=\beta$ follows from a suitable limiting process.

(6) Apply Lemma 2.1 (7) with $r^{2}$ instead of $r$. It is easy to check the formula

$$
\sum_{n=1}^{\infty} \frac{n+\alpha}{n} r^{2 n}=\frac{1}{1-r^{2}}+\alpha \log \frac{1}{1-r^{2}}
$$

It is noteworthy that the above formulae of various series in the proof of the corollary are valid in general regardless of the parameter conditions.

We remark that

$$
A_{0}=\int_{0}^{1} \frac{\log (1 / t)}{1-t} d t=\psi^{\prime}(1)=\sum_{n=1}^{\infty} \frac{1}{n^{2}}=\zeta(2)=\frac{\pi^{2}}{6} .
$$


Therefore, we have the Duren-Leung inequality (2.1) as the limiting case as $\alpha \rightarrow 0$ in (2). Also, we should confess that an application of Lemma 2.1 (4) could not be included in the corollary due to difficulty of evaluation of infinite series of the form

$$
\sum_{n=1}^{\infty} \frac{1}{n\left(n^{2}+a n+b\right)}
$$

when $a^{2}-4 b<0$. We add a couple of further consequences of Theorem 1.1.

Corollary 2.3. (1) $\sum_{n=1}^{\infty} \frac{n^{2}}{(n+1)^{3}}\left|\gamma_{n}\right|^{2} \leq \zeta(3)-1$,

(2) $\sum_{n=1}^{\infty} \frac{n}{(n+1)^{3}}\left|\gamma_{n}\right|^{2} \leq \frac{1}{6}\left(18-\pi^{2}-6 \zeta(3)\right)$.

Proof. (1) follows from the fact that $\varphi(x)=x /(x+1)^{3}$ is convex on $1 \leq x<+\infty$. (2) follows also from the convexity of $\varphi(x)=1 /(x+1)^{3}$ and the computation

$$
\sum_{n=1}^{\infty} \frac{1}{n(n+1)^{3}}=\sum_{n=1}^{\infty}\left(\frac{1}{n(n+1)^{2}}-\frac{1}{(n+1)^{3}}\right)=C_{1,1}-(\zeta(3)-1) .
$$

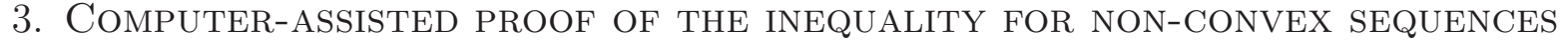

In the first section, we presented an inequality of the logarithmic coefficients $\gamma_{n}$ for a convex sequence $p_{n}$. The inequality may hold even if $p_{n}$ is not convex; namely, some of $\lambda_{n}=p_{n}-2 p_{n+1}+p_{n+2}$ are negative. We review the idea due to Roth [15] and then reformulate it in a convenient form so that one can check the conditions by using computers.

We recall the proof of the Lebedev-Milin conjecture (1.2) by following FitzGerald and Pommerenke [7]. Fix $n \geq 1$. The key idea is to consider the de Branges system of linear ODE:

$$
\tau_{n, k}(t)-\tau_{n, k+1}(t)=-\frac{\tau_{n, k}^{\prime}(t)}{k}-\frac{\tau_{n, k+1}^{\prime}(t)}{k+1}, \quad \tau_{n, k}(0)=n-k+1
$$

for $k=1,2, \ldots, n$, where we put $\tau_{n, n+1}(t) \equiv 0$. With the aid of Löwner chains, we can see that (1.2) follows from the inequalities $\tau_{n, k}^{\prime}(t)<0, t>0, k=1,2, \ldots, n$. See [7] for details. It is known that $\tau_{n, k}^{\prime}(t)$ can be expressed in terms of Jacobi polynomials (see [7, $(2.3)])$ :

$$
\tau_{n, k}^{\prime}(t)=-k e^{-k t} \sum_{j=0}^{n-k} P_{j}^{(2 k, 0)}\left(1-2 e^{-t}\right) .
$$

Here, Jacobi polynomials are defined, for instance, by Rodrigues' formula

$$
P_{j}^{(\alpha, \beta)}(x)=\frac{(-1)^{n}}{2^{n} n !}(1-x)^{-\alpha}(1+x)^{-\beta} \frac{d}{d x}\left[(1-x)^{\alpha}(1+x)^{\beta}\left(1-x^{2}\right)^{n}\right] .
$$

The Askey-Gasper inequality was a key step to confirm $\tau_{n, k}^{\prime}(t)<0$. 
Roth [15] observed that the same idea works for the inequality (1.7). Namely, consider the solution to the initial value problem

$$
\tau_{k}(t)-\tau_{k+1}(t)=-\frac{\tau_{k}^{\prime}(t)}{k}-\frac{\tau_{k+1}^{\prime}(t)}{k+1}, \quad \tau_{k}(0)=\mu_{k}
$$

for $k=1,2, \ldots, N$, where $\tau_{N+1}=0$ and

$$
\mu_{k}=\sum_{j=1}^{N-k+1} j \lambda_{j+k-1}=\sum_{n=k}^{N} \lambda_{n}(n-k+1) .
$$

If the condition

$$
\tau_{k}^{\prime}(t)<0 \text { for } t>0, k=1,2, \ldots, N,
$$

holds, then (1.7) can be deduced in the same way as in [7] (see [15] for details). When $p_{n}=n /(n+1)^{2}$ and $\lambda_{n}=p_{n}-2 p_{n+1}+p_{n+2}$, by solving the differential equations, Roth [15] showed that the condition (3.3) holds for $N=5$.

We take now a slightly different approach below. In view of the form of (1.7), we see that $\tau_{k}$ can be described in terms of the original $\tau_{n, k}$ 's. Indeed, we have

$$
\tau_{k}=\sum_{n=k}^{N} \lambda_{n} \tau_{n, k}
$$

Therefore, by (3.1), $\tau_{k}^{\prime}$ can be expressed in terms of Jacobi polynomials:

$$
\tau_{k}^{\prime}(t)=-k e^{-k t} \sum_{n=k}^{N} \lambda_{n} \sum_{j=0}^{n-k} P_{j}^{(2 k, 0)}\left(1-2 e^{-t}\right)=-k e^{-k t} \sum_{j=0}^{N-k} \nu_{k, j} P_{j}^{(2 k, 0)}\left(1-2 e^{-t}\right),
$$

where

$$
\nu_{k, j}=\sum_{n=j+k}^{N} \lambda_{n}=\lambda_{j+k}+\lambda_{j+k+1}+\cdots+\lambda_{N}=q_{j+k}-q_{N+1} .
$$

We can now summarize these observations as the following theorem.

Theorem 3.1. Let $p_{n}, n=1,2,3, \ldots$, be a sequence of non-negative numbers and set $q_{n}=p_{n}-p_{n+1}$ and $\lambda_{n}=q_{n}-q_{n+1}$. Suppose that there exists a number $N \geq 1$ satisfying the following three conditions:

(0) $p_{N+1}>0$,

(i) $\lambda_{n} \geq 0$ for $n>N$,

(ii) $Q_{k}(x)=\sum_{j=0}^{N-k} \nu_{j+k} P_{j}^{(2 k, 0)}(x)>0$ for $-1<x<1$ and $k=1,2, \ldots, N$, where $\nu_{m}=q_{m}-q_{N+1}$.

Then the inequality

$$
\sum_{n=1}^{\infty} n p_{n}\left|\gamma_{n}\right|^{2} \leq \sum_{n=1}^{\infty} \frac{p_{n}}{n}
$$

holds. Here, equality holds precisely when $f$ is a rotation of the Koebe function $z /(1-z)^{2}$. 
As an example, let us look at the case of Roth [15]. Let $p_{n}=n /(n+1)^{2}$. Then $\lambda_{1}=-1 / 144<0$ but $\lambda_{n}>0$ for $n>1$. Take $N=5$ and compute $Q_{k}, k=1,2,3,4,5$, as follows:

$$
\begin{aligned}
& Q_{1}(x)=\frac{153191+313428 x+443802 x^{2}+517076 x^{3}+249375 x^{4}}{5644800} \\
& Q_{2}(x)=\frac{38929+77359 x+82447 x^{2}+35625 x^{3}}{705600} \\
& Q_{3}(x)=\frac{139643+218986 x+106875 x^{2}}{2822400} \\
& Q_{4}(x)=\frac{15171+11875 x}{705600} \\
& Q_{5}(x)=\frac{95}{28224} .
\end{aligned}
$$

By numerical computations, we can check that $Q_{k}(x)$ has no roots on the interval $(-1,1)$. Hence, we verified the Roth inequality [15]

$$
\sum_{n=1}^{\infty} \frac{n^{2}}{(n+1)^{2}}\left|\gamma_{n}\right|^{2} \leq \frac{\pi^{2}}{6}-1
$$

It is not necessarily easy to check condition (ii) in the theorem. Indeed, we have no general idea about how large $N$ should be chosen. Therefore, the following necessary condition is useful in practical tests.

Proposition 3.2. Under the hypothesis of Theorem [3.1, a necessary condition for (3.1) is

$$
v_{k}=v_{k, N}=\sum_{j=0}^{[(N-k) / 2]} \lambda_{k+2 j}=\lambda_{k}+\lambda_{k+2}+\lambda_{k+4}+\cdots+\lambda_{N^{\prime}} \geq 0, \quad k=1,2, \ldots, N,
$$

where $N^{\prime}=N$ if $N-k$ is even and $N^{\prime}=N-1$ if $N-k$ is odd.

Proof. For (3.1), the condition $\tau_{k}^{\prime}(0) \leq 0$ is necessary. It is noted in [7, p. 685] that $\tau_{n, k}^{\prime}(0)=-k$ if $n-k$ is even and $\tau_{n, k}^{\prime}(0)=0$ if $n-k$ is odd. By (3.4), we have

$$
\tau_{k}^{\prime}(0)=\sum_{n=k}^{N} \lambda_{n} \tau_{n, k}^{\prime}(0)=-k\left(\lambda_{k}+\lambda_{k+2}+\cdots+\lambda_{N^{\prime}}\right)=-k v_{k} .
$$

Thus we have the condition $v_{k} \geq 0$.

For instance,

$$
\begin{aligned}
& v_{1,1}=\lambda_{1}, \\
& v_{1,2}=\lambda_{1}, v_{2,2}=\lambda_{2}, \\
& v_{1,3}=\lambda_{1}+\lambda_{3}, v_{2,3}=\lambda_{2}, v_{3,3}=\lambda_{3}, \\
& v_{1,4}=\lambda_{1}+\lambda_{3}, \quad v_{2,4}=\lambda_{2}+\lambda_{4}, v_{3,4}=\lambda_{3}, v_{4,4}=\lambda_{4} .
\end{aligned}
$$

In particular, we observe that the choice $N \leq 2$ does not work for Theorem 3.1 when $\lambda_{1}<0$. 
Remark. Unfortunately, the condition in the above proposition is not necessarily sufficient. Let $x_{k}(t)=\tau_{k}(t) / k$ for $k=1,2, \ldots, N$. Then the system of ODE (3.2) turns to

$$
x_{k}^{\prime}=-k x_{k}+2(k+1) x_{k+1}-2(k+2) x_{k+2}+\cdots+(-1)^{N-k+1} 2 N x_{N}, \quad x_{k}(0)=\mu_{k} / k
$$

for $k=1,2, \ldots, N$. Introducing the column vector $\boldsymbol{x}=\left(x_{1}, \ldots, x_{N}\right)^{\mathrm{T}}$, the system can be expressed by $\boldsymbol{x}^{\prime}=A_{N} \boldsymbol{x}$ for the $N \times N$ matrix $A_{N}$ corresponding to the above equation. For example,

$$
A_{2}=\left(\begin{array}{cc}
-1 & 4 \\
0 & -2
\end{array}\right), \quad A_{3}=\left(\begin{array}{ccc}
-1 & 4 & -6 \\
0 & -2 & 6 \\
0 & 0 & -3
\end{array}\right)
$$

Letting $\boldsymbol{x}_{0}$ be the initial vector at $t=0$, the solution can be given by $\boldsymbol{x}=e^{t A_{N}} \boldsymbol{x}_{0}$ and thus $\boldsymbol{x}^{\prime}=e^{t A_{N}} A_{N} \boldsymbol{x}_{0}$. In our case, $A_{N} \boldsymbol{x}_{0}=-{ }^{\mathrm{T}}\left(v_{1}, \ldots, v_{N}\right)$, where $v_{k}$ are as in Proposition 3.2. Simple computations give us

$$
e^{t A_{2}}=\left(\begin{array}{cc}
e^{-t} & 4 e^{-t}\left(1-e^{-t}\right) \\
0 & e^{-2 t}
\end{array}\right), \quad e^{t A_{3}}=\left(\begin{array}{ccc}
e^{-t} & 4 e^{-t}\left(1-e^{-t}\right) & 3 e^{-t}\left(1-e^{-t}\right)\left(3-5 e^{-t}\right) \\
0 & e^{-2 t} & 6 e^{-2 t}\left(1-e^{-t}\right) \\
0 & 0 & e^{-3 t}
\end{array}\right) .
$$

We observe that the entry $3 e^{-t}\left(1-e^{-t}\right)\left(3-5 e^{-t}\right)$ of $e^{t A_{3}}$ takes negative values when $t>0$ is small enough. If $\boldsymbol{v}=\left(v_{1}, v_{2}, v_{3}\right)^{\mathrm{T}}$ is very close to $(0,0,1)^{\mathrm{T}}$, then the first entry of $\boldsymbol{x}^{\prime}(t)=e^{t A_{3}} A_{3} \boldsymbol{v}$ will take negative values even if $v_{k}>0$ are satisfied.

As an example, we consider the sequence

$$
p_{n}=\frac{n}{n^{2}+\alpha^{2}} \quad(n=1,2,3, \ldots)
$$

for $\alpha>0$, which appears in Corollary 2.2 (2). Put $q_{n}=p_{n}-p_{n+1}$ and $\lambda_{n}=q_{n}-q_{n+1}$ as before. By Lemma 2.1 and its proof, we see that the sequence $p_{n}$ is convex if and only if $\alpha \leq 1$. It might be an interesting problem to find the largest value $\alpha$ so that the inequality

$$
\sum_{n=1}^{\infty} \frac{n^{2}}{n^{2}+\alpha^{2}}\left|\gamma_{n}\right|^{2} \leq \sum_{n=1}^{\infty} \frac{1}{n^{2}+\alpha^{2}}=\frac{\pi \alpha \operatorname{coth} \pi \alpha-1}{2 \alpha^{2}}
$$

holds for the logarithmic coefficients $\gamma_{n}$ of every function $f \in \mathcal{S}$. For simplicity, put $b=\alpha^{2}>1$. Then $\lambda_{1}<0$ but $\lambda_{2} \geq 0$ if $b \leq 8 / 3$. As we saw, we should choose $N \geq 3$. When $N=3$, we compute

$$
v_{1,3}=\lambda_{1}+\lambda_{3}=\frac{12\left(440-317 b-40 b^{2}-3 b^{3}\right)}{(1+b)\left(2^{2}+b\right)\left(3^{2}+b\right)\left(4^{2}+b\right)\left(5^{2}+b\right)} .
$$

Therefore, $b \leq b_{0}$ is necessary and sufficient for $v_{1,3} \geq 0$, where $b_{0}=1.1925184 \cdots$ is the unique real solution to the equation $440-317 b-40 b^{2}-3 b^{3}=0$. In this case, $v_{2,3}=\lambda_{2}>0, v_{3,3}=\lambda_{3}>0$. A numerical computation tells us that the polynomial $Q_{1}(x)$ in Theorem 3.1 with $N=3$ and $b=b_{0}$ assumes a negative value on $-1<x<1$, see Figure 1. Therefore, the condition in Proposition 3.2 is, indeed, not sufficient for condition (ii) to hold in the theorem. On the other hand, numerical experiments suggest that $Q_{1}(x)>0$ on $-1<x<1$ for $b \leq 1.19245$. Other conditions $Q_{2}(x)>0$ and $Q_{3}(x)>0$ can be checked more easily. Thus, in this case, the inequality (3.6) holds for $b \leq 1.19245$. 


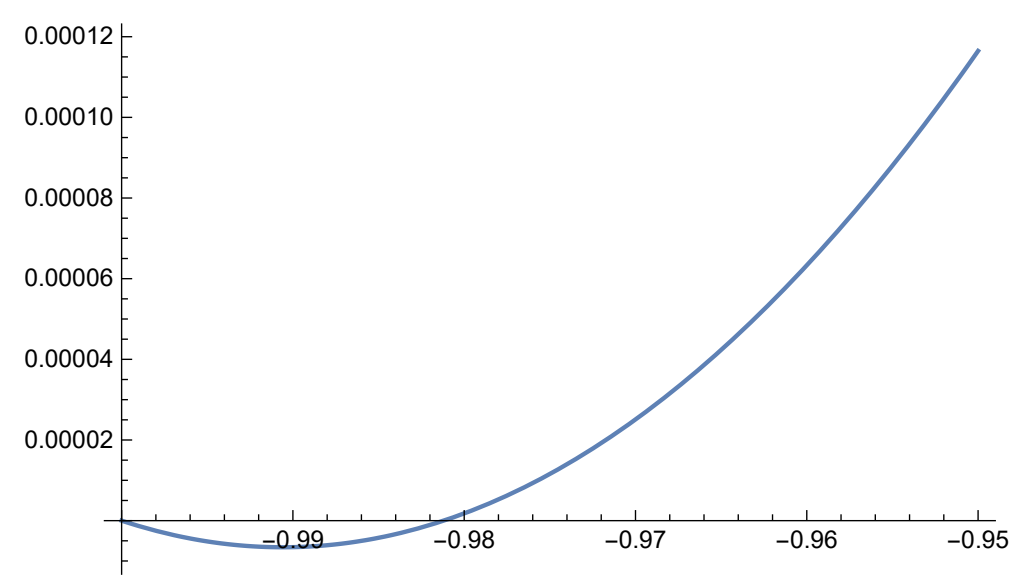

FiguRE 1. The graph of the polynomial $Q_{1}(x)$ for $b=b_{0}$

Letting $N=9$, we can show the following result by using this strategy with the aid of computer.

Theorem 3.3. For the logarithmic coefficients $\gamma_{n}$ of a function $f \in \mathcal{S}$, the inequality

$$
\sum_{n=1}^{\infty} \frac{n^{2}}{n^{2}+4 / 3}\left|\gamma_{n}\right|^{2} \leq B_{2 / \sqrt{3}}=\frac{(2 \pi / \sqrt{3}) \operatorname{coth}(2 \pi / \sqrt{3})-1}{8 / 3}=0.98727 \cdots
$$

holds, where the inequality is strict unless $f$ is a rotation of the Koebe function.

Proof. Let $\varphi(x)=x /\left(x^{2}+4 / 3\right)$ and $p_{n}=\varphi(n)$. Since

$$
\varphi^{\prime \prime}(x)=\frac{54 x(x+2)(x-2)}{\left(3 x^{2}+4\right)^{2}}
$$

we find that $\varphi(x)$ is convex on $2 \leq x<+\infty$. Note that $p_{1}-2 p_{2}+p_{3}=-27 / 868<0$. We computed the polynomials $Q_{k}(x)(k=1,2, \ldots, 9)$ in Theorem 3.1 with $N=9$ by using Mathematica as shown in Appendix. By numerical computations, we found that $Q_{k}(x)$ has no real roots for each odd $k$ and that $Q_{k}(x)$ has only one real root, which is less than -1 , for each even $k$. Thus we confirmed numerically that $Q_{k}(x)>0$ for $-1<x<1$ and $k=1, \ldots, 9$. We now apply Theorem 3.1 to get the assertion.

In a similar way, we can show the following result, which will be used in the next section. Its proof will also given in Appendix.

Theorem 3.4. Let $\beta=1 / 20$. For the logarithmic coefficients $\gamma_{n}$ of a function $f \in \mathcal{S}$, the sharp inequality

$$
\sum_{n=1}^{\infty} \frac{n^{3}\left|\gamma_{n}\right|^{2}}{(n+1)^{2}(n+\beta)} \leq E_{1, \beta}=\frac{20}{19^{2}}\left(1-\gamma-\psi\left(\frac{21}{20}\right)\right)-\frac{20}{19}\left(\frac{\pi^{2}}{6}-1\right)=0.62787 \cdots
$$

holds. 
Proof. Let $\varphi(x)=x^{2} /\left[(x+1)^{2}(x+\beta)\right]$ with $\beta=1 / 20$ and $p_{n}=\varphi(n)$. Then

$$
\varphi^{\prime \prime}(x)=\frac{2\left\{x^{2}(x-3)(x+1)+(3-6 \beta) x^{2}-2 \beta^{2} x+\beta^{2}\right\}}{(x+1)^{4}(\beta+x)^{3}}
$$

is positive for $x \geq 3$. In this case, indeed, we have

$$
\lambda_{1}=-\frac{6985}{630252} \quad \text { and } \quad \lambda_{2}=\frac{12103}{2025810}
$$

for $\lambda_{n}=p_{n}-2 p_{n+1}+p_{n+2}$. We take $N=9$ and compute $Q_{k}(x)(k=1, \ldots, 9)$ as shown in Appendix. By numerical computations, as in the previous case, $Q_{k}(x)$ has no real roots for each odd $k$ and $Q_{k}(x)$ has only one real root, which is less than -1 , for each even $k$. Thus we confirm the assertion in the same way as the previous theorem.

\section{Applications}

Our next result is related to a transform $h_{f}$ of $f \in \mathcal{S}$ introduced by Danikas and Ruscheweyh [4]:

$$
h_{f}(z):=\int_{0}^{z} \frac{t f^{\prime}(t)}{f(t)} d t
$$

It was conjectured in [4] that the transform $h_{f} \in \mathcal{S}$ for each $f \in \mathcal{S}$. This conjecture remains open. Roth [15] applied his inequality (3.5) to obtain the sharp $H^{2}$ norm estimate of $h_{f}$ for $f \in \mathcal{S}$. We now introduce the class

$$
\mathcal{U}=\left\{f \in \mathcal{A}:\left|U_{f}(z)\right|<1 \text { for } z \in \mathbb{D}\right\},
$$

where

$$
U_{f}(z)=f^{\prime}(z)\left(\frac{z}{f(z)}\right)^{2}-1, \quad z \in \mathbb{D} .
$$

It is known that $\mathcal{U} \subset \mathcal{S}$. See [3] and also [8, 11, 12, and the references therein. We will say that $f \in \mathcal{U}$ on $|z|<r$ if $f_{r}(z)=f(r z) / r$ belongs to $\mathcal{U}$. Several generalizations of the class $\mathcal{U}$ were investigated in the literature. Among them, the following result was proved in [13].

Theorem A ([13, Thoerem 4]). Let $f \in \mathcal{S}, b=\left|f^{\prime \prime}(0)\right| / 2$ !, and let $H$ be defined by the quotient

$$
H(z)=\frac{z^{2}}{h_{f}(z)} .
$$

Then $H \in \mathcal{U}$ on the disk $|z|<r_{1}(b)$. Here, $r_{1}(b) \geq r_{1}(0) \approx 0.557666$ is the root of the equation

$$
\left(\frac{2 \pi^{2}}{3}-4-\frac{b^{2}}{4}\right) r^{4}\left(1+r^{2}\right)=\left(1-r^{2}\right)^{3}
$$

in $0<r<1$ for $b \in[0,2]$.

The proof of this theorem is based on the Roth inequality (3.5). It is almost the optimal choice but there is still room to improve a little as follows. 
Theorem 4.1. Let $f \in \mathcal{S}, b=\left|f^{\prime \prime}(0)\right| / 2$ !, and let $H$ be defined by (4.2). Then $H \in \mathcal{U}$ in the disk $|z|<r_{2}(b)$, where $r_{2}(b) \geq r_{2}(0) \approx 0.558509$ is the solution of the equation

$$
\log \frac{1}{1-r^{2}}+\frac{-r^{2}+23 r^{4}+18 r^{6}}{\left(1-r^{2}\right)^{3}}=\frac{20}{4 E_{1,1 / 20}-5 b^{2} / 84}
$$

in $0<r<1$ for $b \in[0,2]$ and $E_{1,1 / 20}$ is the constant given in Theorem 3.4.

The method of the proof is along the line of [13] but based on Theorem 3.4 instead of the Roth inequality.

Proof of Theorem 4.1. First we note the expression

$$
h_{f}(z)=\int_{0}^{z}\left(1+t\left(\log \frac{f(t)}{t}\right)^{\prime}\right) d t=z+2 \sum_{n=2}^{\infty} \frac{n-1}{n} \gamma_{n-1} z^{n},
$$

where $\gamma_{n}(n \geq 1)$ denote the logarithmic coefficients of $f \in \mathcal{S}$ defined by (1.1). We also have

$$
\frac{z}{H(z)}=1+2 \sum_{n=1}^{\infty} \frac{n}{n+1} \gamma_{n} z^{n}
$$

and $2\left|\gamma_{1}\right|=\left|f^{\prime \prime}(0)\right| / 2=b$. By the forms of $U_{H}(z)$ and $H$, we compute

$$
U_{H}(z)=-z^{2}\left(\frac{1}{H(z)}-\frac{1}{z}\right)^{\prime}=-z\left(\frac{z}{H(z)}\right)^{\prime}+\frac{z}{H(z)}-1=-2 \sum_{n=2}^{\infty} \frac{(n-1) n}{n+1} \gamma_{n} z^{n} .
$$

Letting $r=|z|<1$, we estimate with the help of the Cauchy-Schwarz inequality in addition to Theorem 3.4 as follows:

$$
\begin{aligned}
\left|U_{H}(z)\right| & \leq 2 \sum_{n=2}^{\infty} \frac{(n-1) n}{n+1}\left|\gamma_{n}\right||z|^{n} \\
& \leq 2\left(\sum_{n=2}^{\infty} \frac{n^{3}}{(n+1)^{2}(n+\beta)}\left|\gamma_{n}\right|^{2}\right)^{1 / 2}\left(\sum_{n=2}^{\infty} \frac{(n-1)^{2}(n+\beta)}{n} r^{2 n}\right)^{1 / 2} \\
& \leq 2\left(E_{1, \beta}-\frac{5\left|\gamma_{1}\right|^{2}}{84}\right)^{1 / 2}\left(\frac{-r^{2}+23 r^{4}+18 r^{6}}{20\left(1-r^{2}\right)^{3}}-\frac{1}{20} \log \left(1-r^{2}\right)\right)^{1 / 2}
\end{aligned}
$$

which is less than 1 whenever,

$$
\log \frac{1}{1-r^{2}}+\frac{-r^{2}+23 r^{4}+18 r^{6}}{\left(1-r^{2}\right)^{3}}<\frac{20}{4 E_{1,1 / 20}-5 b^{2} / 84} .
$$

Note that the left-hand quantity is increasing from 0 to $+\infty$ when $r$ moves from 0 to 1 so that the root $r_{2}(b)$ of the equation in the statement is an increasing function of $b$ on the interval $[0,2]$.

By using Mathematica, we made graphs of the functions $r=r_{1}(b)$ and $r=r_{2}(b)$ and a graph of the difference $r_{2}(b)-r_{1}(b)$ in Figure 2 .

In a paper [14], analytic and geometric properties of the function $P_{f}(z)=f(z) / f^{\prime}(z)$ are studied for $f \in \mathcal{S}$. Let us look at the following result in the paper. 


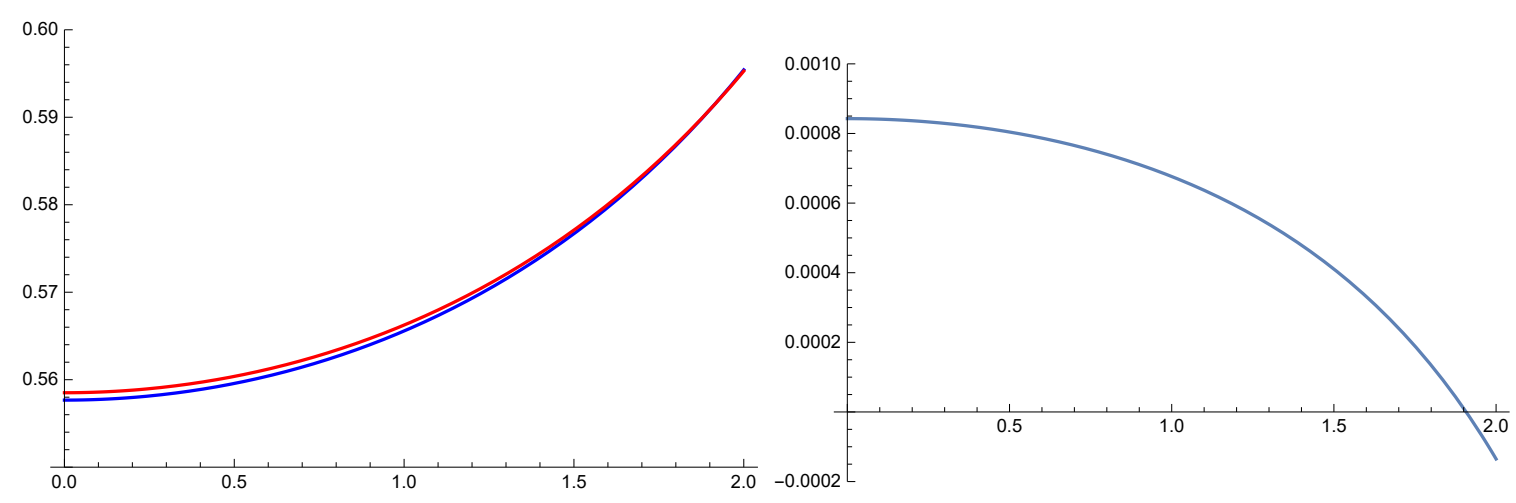

Figure 2. Left: the graphs of $r_{1}(b)$ (blue colored) and $r_{2}(b)$ (red colored), Right: the graph of the difference $r_{2}(b)-r_{1}(b)$

Theorem B ([14, Theorem 3.16]). Let $f \in \mathcal{S}$ and $b=\left|f^{\prime \prime}(0)\right| / 2$ !. Then $P_{f} \in \mathcal{U}$ on the disk $|z|<r_{3}(b)$, where $r=r_{3}(b) \geq r_{3}(0) \approx 0.360794$ is the root of the equation

$$
\left(\frac{2 \pi^{2}}{3}-4-\frac{b^{2}}{4}\right) r^{4}\left(r^{6}-5 r^{4}+19 r^{2}+9\right)=\left(1-r^{2}\right)^{5}
$$

in $0<r<1$ for $b \in[0,2]$. 3.3 .

Their proof relied also on the Roth inequality (3.5). Here, we replace it by Theorem

Theorem 4.2. Let $f \in \mathcal{S}$ and $b=\left|f^{\prime \prime}(0)\right| / 2$ !. Then $P_{f} \in \mathcal{U}$ on the disk $|z|<r_{4}(b)$. Here, $r=r_{4}(b)$ is the solution of the equation

$$
\left(4 B_{2 / \sqrt{3}}-\frac{3 b^{2}}{7}\right) r^{4}\left(r^{6}+2 r^{4}+11 r^{2}+4\right)=\frac{3}{4}\left(1-r^{2}\right)^{5}
$$

in $0<r<1$ for $b \in[0,2]$ and $B_{2 / \sqrt{3}}$ is the constant given in Theorem 3.3. The function $r_{4}(b)$ is increasing in $0 \leq b \leq 2$ and $r_{4}(b) \geq r_{4}(0) \approx 0.362012$.

Proof. Let $F=P_{f}$. Since

$$
\frac{z f^{\prime}(z)}{f(z)}=1+2 \sum_{n=1}^{\infty} n \gamma_{n} z^{n}
$$

we obtain the expressions

$$
\begin{aligned}
U_{F}(z) & =F^{\prime}(z)\left(\frac{z}{F(z)}\right)^{2}-1=\frac{z f^{\prime}(z)}{f(z)}-z\left(\frac{z f^{\prime}(z)}{F(z)}\right)^{\prime}-1 \\
& =-2 \sum_{n=1}^{\infty} n(n-1) \gamma_{n} z^{n} .
\end{aligned}
$$




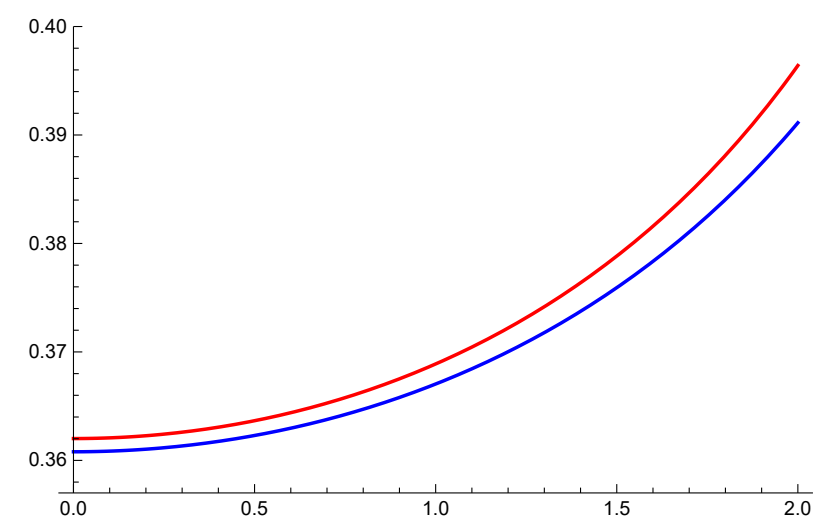

Figure 3. The graphs of $r_{3}(b)$ (blue colored) and $r_{4}(b)$ (red colored)

Hence, as in the proof of Theorem 4.1, we estimate

$$
\begin{aligned}
\left|U_{H}(z)\right| & \leq 2 \sum_{n=2}^{\infty}(n-1) n\left|\gamma_{n}\right||z|^{n} \\
& \leq 2\left(\sum_{n=2}^{\infty} \frac{n^{2}}{n^{2}+4 / 3}\left|\gamma_{n}\right|^{2}\right)^{1 / 2}\left(\sum_{n=2}^{\infty}(n-1)^{2}\left(n^{2}+4 / 3\right) r^{2 n}\right)^{1 / 2} \\
& \leq 2\left(B_{2 / \sqrt{3}}-\frac{3\left|\gamma_{1}\right|^{2}}{7}\right)^{1 / 2}\left(\frac{4 r^{4}\left(r^{6}+2 r^{4}+11 r^{2}+4\right)}{3\left(1-r^{2}\right)^{5}}\right)^{1 / 2}
\end{aligned}
$$

We now see that $\left|U_{F}(z)\right|<1$ as long as

$$
\frac{4 r^{4}\left(r^{6}+2 r^{4}+11 r^{2}+4\right)}{3\left(1-r^{2}\right)^{5}}<\frac{1}{4 B_{2 / \sqrt{3}}-3 b^{2} / 7} .
$$

Now the assertion follows as before.

In Figure 3, we exhibit the graphs of $r_{3}(b)$ and $r_{4}(b)$. 


\section{ApPENDiX}

The polynomials $Q_{k}(x)(k=1,2, \ldots, 9)$ used in the proof of Theorem 3.3 are presented below. We note that by using a suitable command of Mathematica or similar software, we can find all the roots of the following polynomials numerically. In this way, we can check that $Q_{k}$ has no roots on the interval $(-1,1)$ so that $Q_{k}(x)>0$ for $(-1,1)$.

$$
\begin{aligned}
Q_{1}(x) & =\frac{1136025 x^{8}}{3570176}+\frac{387585 x^{7}}{575456}+\frac{285789141 x^{6}}{943419008}-\frac{103110975 x^{5}}{825491632}+\frac{34505962335 x^{4}}{1043421422848} \\
& +\frac{380568045735 x^{3}}{1695559812128}+\frac{46758786465915 x^{2}}{210249416703872}+\frac{2558807811009 x}{13140588543992}+\frac{77049161884395}{840997666815488} \\
Q_{2}(x)= & \frac{103275 x^{7}}{223136}+\frac{3957741 x^{6}}{3123904}+\frac{9202167 x^{5}}{7370461}+\frac{108476415 x^{4}}{173787712}+\frac{7350044085 x^{3}}{18632525408} \\
& +\frac{1429990103205 x^{2}}{3391119624256}+\frac{9108847966527 x}{26281177087984}+\frac{19344079210563}{105124708351936}, \\
Q_{3}(x)= & \frac{240975 x^{6}}{446272}+\frac{2618811 x^{5}}{1561952}+\frac{984379149 x^{4}}{471709504}+\frac{1223639757 x^{3}}{825491632}+\frac{222176774097 x^{2}}{260855355712} \\
& +\frac{821182609953 x}{1695559812128}+\frac{20630684258217}{105124708351936}, \\
Q_{4}(x)= & \frac{722925 x^{5}}{1450384}+\frac{32587515 x^{4}}{20305376}+\frac{805347441 x^{3}}{383263972}+\frac{16309308609 x^{2}}{10731391216}+\frac{607240980387 x}{847779906064} \\
+ & \frac{357105897585}{1695559812128}, \\
Q_{5}(x) & =\frac{516375 x^{4}}{1450384}+\frac{696195 x^{3}}{634543}+\frac{1015598331 x^{2}}{766527944}+\frac{1064477133 x}{1341423902}+\frac{188723150883}{847779906064}, \\
Q_{6}(x)= & \frac{34425 x^{3}}{181298}+\frac{9077265 x^{2}}{17767204}+\frac{326148282 x}{670711951}+\frac{463541805}{2682847804}, \\
Q_{7}(x)= & \frac{103275 x^{2}}{1450384}+\frac{5178573 x}{35534408}+\frac{857191005}{10731391216}, \\
Q_{8}(x)= & \frac{6075 x}{362596}+\frac{684531}{35534408}, \\
Q_{9}(x)= & \frac{675}{362596} .
\end{aligned}
$$


The polynomials $Q_{k}(x)(k=1,2, \ldots, 9)$ used in the proof of Theorem 3.4 are presented below.

$$
\begin{aligned}
& Q_{1}(x)=\frac{62987827 x^{8}}{341496320}+\frac{330128990251 x^{7}}{860326246080}+\frac{158362220519819 x^{6}}{1016659815367680} \\
& -\frac{10529204214766063 x^{5}}{107935383731535360}-\frac{530240051345429 x^{4}}{93380566898691072}+\frac{541351055064272599 x^{3}}{5450736878442535680} \\
& +\frac{1284708860080692110137 x^{2}}{14629777781739765765120}+\frac{1777709878968276897929 x}{27264585865969563471360} \\
& +\frac{1284524775560504080639}{43889333345219297295360} \\
& Q_{2}(x)=\frac{62987827 x^{7}}{234778720}+\frac{5390982010279 x^{6}}{7435676841120}+\frac{1932000965815939 x^{5}}{2795814492261120} \\
& +\frac{110402609517184133 x^{4}}{356186766314066688}+\frac{438453616394854873 x^{3}}{2569633099837195392}+\frac{16605230695470458161 x^{2}}{89937158494301838720} \\
& +\frac{1581160573987373624339 x}{10972333336304824323840}+\frac{30873002384858864057389}{449865666788497797277440}, \\
& Q_{3}(x)=\frac{440914789 x^{6}}{1408672320}+\frac{2383515042599 x^{5}}{2478558947040}+\frac{3620224806455 x^{4}}{3089297781504} \\
& +\frac{70911808727134489 x^{3}}{89046691578516672}+\frac{852605666350380625 x^{2}}{1998603522095596416}+\frac{10225388053815434921 x}{44968579247150919360} \\
& +\frac{942885972184285561567}{10972333336304824323840} \\
& Q_{4}(x)=\frac{440914789 x^{5}}{1526061680}+\frac{8910108245315 x^{4}}{9666379893456}+\frac{98087517932455 x^{3}}{82603609998624} \\
& +\frac{20630163482121289 x^{2}}{24735192105143520}+\frac{104078041421486463 x}{277583822513277280}+\frac{2331963676119479803}{22484289623575459680}, \\
& Q_{5}(x)=\frac{62987827 x^{4}}{305212336}+\frac{1524238766119 x^{3}}{2416594973364}+\frac{105039968099029 x^{2}}{139790724613056} \\
& +\frac{5760631998672587 x}{13095101702723040}+\frac{486393597309120707}{4088052658831901760} \\
& Q_{6}(x)=\frac{62987827 x^{3}}{572273130}+\frac{318698320009 x^{2}}{1084369539330}+\frac{4395382293406319 x}{15901194924735120}+\frac{10724868335939851}{111308364473145840}, \\
& Q_{7}(x)=\frac{62987827 x^{2}}{1526061680}+\frac{14188797597869 x}{169161648135480}+\frac{120529899535861}{2650199154122520}, \\
& Q_{8}(x)=\frac{62987827 x}{6485762140}+\frac{938138865611}{84580824067740}, \\
& Q_{9}(x)=\frac{62987827}{58371859260} \text {. }
\end{aligned}
$$

\section{REFERENCES}

1. M. Abramowitz and I. A. Stegun, Handbook of Mathematical Functions, Dover, 1972.

2. L. V. Ahlfors, Complex Analysis, 3rd ed., McGraw Hill, New York, 1979. 
3. L. A. Aksent'ev, Sufficient conditions for univalence of regular functions (Russian), Izv. Vyš̌. Učebn. Zaved. Matematika 1958 (1958), no. 3 (4), 3-7.

4. N. Danikas and St. Ruscheweyh, Semi-convex hulls of analytic functions in the unit disk, Analysis 4 (1999), 309-318.

5. L. de Branges, A proof of the Bieberbach conjecture, Acta Math. 154 (1985), 137-152.

6. P. L. Duren and Y. J. Leung, Logarithmic coefficients of univalent functions, J. Anal. Math. 36 (1979), 36-43.

7. C. H. FitzGerald and Ch. Pommerenke, The de Branges theorem on univalent functions, Trans. Amer. Math. Soc. 290 (1985), 683-690.

8. R. Fournier and S. Ponnusamy, A class of locally univalent functions defined by a differential inequality, Complex Var. Elliptic Equ. 52 (2006), 1-8.

9. I. M. Milin, Some applications of theorems on logarithmic coefficients (Russian), Sibirsk. Mat. Zh. 32 (1991), 87-98, 220, English translation in Siberian Math. J. 32 (1991), 69-78.

10. I. M. Milin and A. Z. Grinshpan, Logarithmic coefficients means of univalent functions, Complex Var. 7 (1986), 139-147.

11. M. Obradović and S. Ponnusamy, New criteria and distortion theorems for univalent functions, Complex Variables Theory Appl. 44 (2001), 173-191.

12. M. Obradović and S. Ponnusamy, Univalence and starlikeness of certain integral transforms defined by convolution of analytic functions, J. Math. Anal. Appl. 336 (2007), 758-767.

13. M. Obradović and S. Ponnusamy, Univalence of quotient of analytic functions, Appl. Math. Comput. 247 (2014), 689-694.

14. M. Obradović, S. Ponnusamy, and K.-J. Wirths, Where is $f(z) / f^{\prime}(z)$ univalent?, J. Anal. 22 (2014), 131-143.

15. O. Roth, A sharp inequality for the logarithmic coefficients of univalent functions, Proc. Amer. Math. Soc. 135 (2007), 2051-2054.

16. S. M. Zemyan, Estimates of logarithmic coeffients of univalent funcions, Internat. J. Math. Math. Sci. 16 (1993), 311-318.

S. Ponnusamy, Department of Mathematics, Indian Institute of Technology Madras, Chennai-600 036, India.

E-mail address: samy@iitm.ac.in

Graduate School of Information Sciences, Tohoku University, Aoba-ku, Sendai 9808579, JAPAN

E-mail address: sugawa@math.is.tohoku.ac.jp 